\title{
Intuitionistic Neutrosphic Soft Set over Rings
}

\author{
Said Broumi ${ }^{1, *}$, Florentin Smarandache ${ }^{2}$, Pabitra Kumar Maji ${ }^{3}$ \\ ${ }^{1}$ Administrator of Faculty of Arts and Humanities, Hay El Baraka Ben M'sik Casablanca B.P. 7951, Hassan II University \\ Mohammedia-Casablanca, Morocco \\ ${ }^{2}$ Department of Mathematics, University of New Mexico, 705 Gurley Avenue, Gallup, NM 87301, USA \\ ${ }^{3}$ Department of Mathematics, B. C. College, Asansol, Dist.- Burdwan, West Bengal, 713304, INDIA \\ *Corresponding Author: broumisaid78@gmail.com
}

Copyright $@ 2014$ Horizon Research Publishing All rights reserved

\begin{abstract}
S.Broumi and F.Smarandache introduced the concept of intuitionistic neutrosophic soft set as an extension of the soft set theory. In this paper we have applied the concept of intuitionistic neutrosophic soft set to rings theory. The notion of intuitionistic neutrosophic soft set over ring (INSSOR for short) is introduced and their basic properties have been investigated.The definitions of intersection, union, AND, and OR operations over ring (INSSOR) have also been defined. Finally, we have defined the product of two intuitionistic neutrosophic soft set over ring.
\end{abstract}

Keywords Intuitionistic Neutrosphic Soft Set, Intuitionistic Neutrosphic Soft Set over Ring, Soft Set, Neutrosphic Soft Set

\section{Introduction}

The theory of neutrosophic set (NS), which is the generalization of the classical sets, conventional fuzzy set [1], intuitionistic fuzzy set [2] and interval valued fuzzy set [3],was introduced by Samarandache [4]. This concept has recently motivated new research in several directions such as databases [5,6], medical diagnosis problem [7],decision making problem [8],topology [9],control theory [10]and so on. We become handicapped to use fuzzy sets, intuitionistic fuzzy sets or interval valued fuzzy sets when the indeterministic part of uncertain data plays an important role to make a decision. In this context some works can be found in $[11,12,13,14]$.

Another important concept that addresses uncertain information is the soft set theory originated by Molodtsov[15]. This concept is free from the parameterization inadequacy syndrome of fuzzy set theory, rough set theory, probability theory. Molodtsov has successfully applied the soft set theory in many different fields such as smoothness of functions, game theory, operations research, Riemann integration, Perron integration, and probability.
In recent years, soft set theory has been received much attention since its appearance. There are many papers devoted to fuzzify the concept of soft set theory which leads to a series of mathematical models such as fuzzy soft set $[16,17,18,19,20]$, generalized fuzzy soft set $[21,22]$, possibility fuzzy soft set [23] and so on. Thereafter,P.K.Maji and his coworker[24]introduced the notion of intuitionistic fuzzy soft set which is based on a combination of the intuitionistic fuzzy sets and soft set models and studied the properties of intuitionistic fuzzy soft set. Later, a lot of extentions of intuitionistic fuzzy soft are appeared such as generalized intuitionistic fuzzy soft set [25], possibility Intuitionistic fuzzy soft set [26] and so on. Furthermore, few researchers have contributed a lot towards neutrosophication of soft set theory. In [27] P.K.Maji, first proposed a new mathematical model called "neutrosophic soft set" and investigate some properties regarding neutrosophic soft union, neutrosophic soft intersection, complement of a neutrosophic soft set ,De Morgan's laws. In 2013, S.Broumi and F. Smarandache [28]combined the intuitionistic neutrosophic set and soft set which lead to a new mathematical model called" intutionistic neutrosophic soft sets". They studied the notions of intuitionistic neutrosophic soft set union, intuitionistic neutrosophic soft set intersection, complement of intuitionistic neutrosophic soft set and several other properties of intuitionistic neutrosophic soft set along with examples and proofs of certain results. S.Broumi [29]presented the concept of "generalized neutrosophic soft set" by combining the generalized neutrosophic sets[13] and soft set models, studied some properties on it, and presented an application of generalized neutrosophic soft Set in decision making problem.

The algebraic structure of soft set theories has been explored in recent years. In [30], Aktas and Cagman gave a definition of soft groups and compared soft sets to the related concepts of fuzzy sets and rough sets. Sezgin and Atagün [33] defined the notion of normalistic soft groups and corrected some of the problematic cases in paper by Aktas and Cagman [30]. Aygunoglu and Aygun [31] introduced the notion of fuzzy soft groups based on Rosenfeld's approach [32] and studied its properties. In 2010, Acar et al. [34] introduced the 
basic notion of soft rings which are actually a parametrized family of subrings. Ghosh, Binda and Samanta [35] introduced the notion of fuzzy soft rings and fuzzy soft ideals and studied some of its algebraic properties. Inan and Ozturk [36]concurrently studied the notion of fuzzy soft rings and fuzzy soft ideals but they dealt with these concepts in a more detailed manner compared to Ghosh et al.[35]. In 2012, B.P.Varol et al [37]introduced the notion of fuzzy soft ring in different way and studied several of their basic properties. J. Zhan et al[38]introduced soft rings related to fuzzy set theory. G. Selvachandran and A. R. Salleh[39]introduced vague soft rings and vague soft ideals and studied some of their basic properties. Z.Zhang [40] introduced intuitionistic fuzzy soft rings studied the algebraic properties of intuitionistic fuzzy soft ring. Studies of fuzzy soft rings are carried out by several researchers but the notion of neutrosophic soft rings is not studied. So, in this work we first study with the algebraic properties of intuitionistic neutrosophic soft set in ring theory. This paper is organized as follows. In section 2 we gives some known and useful preliminary definitions and notations on soft set theory, neutrosophic set, intuitionistic neutrosophic set, intuitionistic neutrosophic soft set and ring theory. In section 3 we discuss intuitionistic neutrosophic soft set over ring (INSSOR). In section 4 concludes the paper.

\section{Preliminaries}

In this section we recapitulate some relevant definitions viz, soft set, neutrosophic set, intuitionistic neutrosophic set, intuitionistic neutrosophic soft sets, fuzzy subring for better understanding of this article.

\subsection{Definition [15]}

Molodtsov defined the notion of a soft set in the following way: Let $\mathrm{U}$ be an initial universe and $\mathrm{E}$ be a set of parameters. Let $\zeta(\mathrm{U})$ denotes the power set of $\mathrm{U}$ and $\mathrm{A}$ be a non-empty subset of $E$. Then a pair $(P, A)$ is called a soft set over $U$, where $\mathrm{P}$ is a mapping given by $\mathrm{P}: \mathrm{A} \rightarrow \zeta(\mathrm{U})$. In other words, a soft set over $U$ is a parameterized family of subsets of the universe $\mathrm{U}$. For $\varepsilon \in \mathrm{A}, \mathrm{P}(\varepsilon)$ may be considered as the set of $\varepsilon$-approximate elements of the soft set (P, A).

\subsection{Definition [4]}

Let $U$ be an universe of discourse then the neutrosophic set $\mathrm{A}$ is an object having the form $\mathrm{A}=\{<\mathrm{x}$ : $\mathrm{T}_{\mathrm{A}}(\mathrm{x}), \mathrm{I}_{\mathrm{A}}(\mathrm{x}), \mathrm{F}_{\mathrm{A}}(\mathrm{x})>, \mathrm{x} \in \mathrm{U}$, , where the functions $\mathrm{T}, \mathrm{I}, \mathrm{F}$ : $\mathrm{U} \rightarrow]^{-} 0,1^{+}$[define respectively the degree of membership , the degree of indeterminacy, and the degree of non-membership of the element $\mathrm{x} \in \mathrm{X}$ to the set $\mathrm{A}$ with the condition.

$$
-0 \leq \mathrm{T}_{\mathrm{A}}(\mathrm{x})+\mathrm{I}_{\mathrm{A}}(\mathrm{x})+\mathrm{F}_{\mathrm{A}}(\mathrm{x}) \leq 3^{+} .
$$

From philosophical point of view, the neutrosophic set takes the value from real standard or non-standard subsets of $]^{-} 0,1^{+}[\text {.So instead of }]^{-} 0,1^{+}$[we need to take the interval[ 0,1$]$ for technical applications, because $]^{-} 0,1^{+}$[will be difficult to apply in the real applications such as in scientific and engineering problems.

\subsection{Definition [11]}

An element $x$ of $U$ is called significant with respect to neutrosophic set $A$ of $U$ if the degree of truth-membership or falsity-membership or indeterminancy-membership value, i.e., $\mathrm{T}_{A}(\mathrm{x})$ or $\mathrm{I}_{A}(\mathrm{x})$ or $\mathrm{F}_{\mathrm{A}}(\mathrm{x}) \leq 0.5$. Otherwise, we call it insignificant. Also, for neutrosophic set the truth-membership, indeterminacy-membership and falsity-membership all can not be significant. We define an intuitionistic neutrosophic set by

$$
\begin{aligned}
\mathrm{A}=\left\{<\mathrm{x}: \mathrm{T}_{\mathrm{A}}(\mathrm{x}), \mathrm{I}_{\mathrm{A}}(\mathrm{x}), \mathrm{F}_{\mathrm{A}}(\mathrm{x})>, \mathrm{x} \in \mathrm{U}\right\}, \text { where } \\
\min \left\{\mathrm{T}_{\mathrm{A}}(\mathrm{x}), \mathrm{F}_{\mathrm{A}}(\mathrm{x})\right\} \leq 0.5, \\
\min \left\{\mathrm{T}_{\mathrm{A}}(\mathrm{x}), \mathrm{I}_{\mathrm{A}}(\mathrm{x})\right\} \leq 0.5, \\
\min \left\{\mathrm{F}_{\mathrm{A}}(\mathrm{x}), \mathrm{I}_{\mathrm{A}}(\mathrm{x})\right\} \leq 0.5, \text { for all } \mathrm{x} \in \mathrm{U},
\end{aligned}
$$

with the condition $0 \leq \mathrm{T}_{\mathrm{A}}(\mathrm{x})+\mathrm{I}_{\mathrm{A}}(\mathrm{x})+\mathrm{F}_{\mathrm{A}}(\mathrm{x}) \leq 2$.

As an illustration, let us consider the following example.

\subsection{Example}

Assume that the universe of discourse $\mathrm{U}=\left\{\mathrm{x}_{1}, \mathrm{x}_{2}, \mathrm{x}_{3}\right\}$, where $\mathrm{x}_{1}$ characterizes the capability, $\mathrm{x}_{2}$ characterizes the trustworthiness and $\mathrm{x}_{3}$ indicates the prices of the objects. Further, It may be assumed that the values of $\mathrm{x}_{1}, \mathrm{x}_{2}$ and $\mathrm{x}_{3}$ are in $[0,1]$ and they are obtained from some questionnaires of some experts. The experts may impose their opinion in three components viz. the degree of goodness, the degree of indeterminacy and that of poorness to explain the characteristics of the objects. Suppose A is an intuitionistic neutrosophic set ( INS ) of $\mathrm{U}$, such that,

$\left.\mathrm{A}=\left\{<x_{1}, 0.3,0.5,0.4\right\rangle,<x_{2}, 0.4,0.2,0.6\right\rangle,<x_{3}, 0.7$, $0.3,0.5>$, where the degree of goodness of capability is 0.3 , degree of indeterminacy of capability is 0.5 and degree of falsity of capability is 0.4 etc.

\subsection{Definition [28]}

Let $\mathrm{U}$ be an initial universe set and $\mathrm{A} \subset \mathrm{E}$ be a set of parameters. Let $\mathrm{N}(\mathrm{U})$ denotes the set of all intuitionistic neutrosophic sets of $\mathrm{U}$. The collection $(\mathrm{P}, \mathrm{A})$ is termed to be the soft intuitionistic neutrosophic set over $\mathrm{U}$, where $\mathrm{P}$ is a mapping given by $\mathrm{P}: \mathrm{A} \rightarrow \mathrm{N}(\mathrm{U})$.

\subsection{Remark}

We will denote the intuitionistic neutrosophic soft set defined over a universe by INSS.

Let us consider the following example.

\subsection{Example}


Let $\mathrm{U}$ be the set of blouses under consideration and $\mathrm{E}$ is the set of parameters (or qualities). Each parameter is a intuitionistic neutrosophic word or sentence involving intuitionistic neutrosophic words. Consider $\mathrm{E}=\{$ Bright, Cheap, Costly, very costly, Colorful, Cotton, Polystyrene, long sleeve, expensive $\}$. In this case, to define a intuitionistic neutrosophic soft set means to point out Bright blouses, Cheap blouses, Blouses in Cotton and so on. Suppose that, there are five blouses in the universe $U$ given by, $U=\left\{b_{1}, b_{2}, b_{3}, b_{4}, b_{5}\right\}$ and the set of parameters $A=\left\{e_{1}\right.$, $\left.\mathrm{e}_{2}, \mathrm{e}_{3}, \mathrm{e}_{4}\right\}$, where each $\mathrm{e}_{\mathrm{i}}$ is a specific criterion for blouses: $\mathrm{e}_{1}$ stands for 'Bright', $\mathrm{e}_{2}$ stands for 'Cheap',

$\mathrm{e}_{3}$ stands for 'costly',

$\mathrm{e}_{4}$ stands for 'Colorful',

Suppose that,

$\mathrm{P}($ Bright $)=\left\{<\mathrm{b}_{1}, 0.5,0.6,0.3>,<\mathrm{b}_{2}, 0.4,0.7,0.2>,<\mathrm{b}_{3}, 0.6,0\right.$. $\left.2,0.3>,<\mathrm{b}_{4}, 0.7,0.3,0.2>,<\mathrm{b}_{5}, 0.8,0.2,0.3>\right\}$.

$\mathrm{P}($ Cheap $)=\left\{<\mathrm{b}_{1}, 0.6,0.3,0.5\right\rangle,<\mathrm{b}_{2}, 0.7,0.4,0.3>,<\mathrm{b}_{3}, 0.8,0$. $\left.1,0.2>,<\mathrm{b}_{4}, 0.7,0.1,0.3>,<\mathrm{b}_{5}, 0.8,0.3,0.4\right\}$.

$\mathrm{P}($ Costly $)=\left\{<\mathrm{b}_{1}, 0.7,0.4,0.3>,<\mathrm{b}_{2}, 0.6,0.1,0.2>,<\mathrm{b}_{3}, 0.7,0\right.$. $\left.2,0.5>,<\mathrm{b}_{4}, 0.5,0.2,0.6>,<\mathrm{b}_{5}, 0.7,0.3,0.2>\right\}$.

$\mathrm{P}($ Colorful $)=\left\{<\mathrm{b}_{1}, 0.8,0.1,0.4>,<\mathrm{b}_{2}, 0.4,0.2,0.6>,<\mathrm{b}_{3}, 0.3\right.$, $\left.0.6,0.4>,<\mathrm{b}_{4}, 0.4,0.8,0.5>,<\mathrm{b}_{5}, 0.3,0.5,0.7>\right\}$.

\subsection{Definition [28]}

For two intuitionistic neutrosophic soft sets $(\mathrm{P}, \mathrm{A})$ and $(\mathrm{Q}, \mathrm{B})$ over the common universe $\mathrm{U}$. We say that $(\mathrm{P}, \mathrm{A})$ is an intuitionistic neutrosophic soft subset of $(\mathrm{Q}, \mathrm{B})$ if and only if (i) $\mathrm{A} \subset \mathrm{B}$.

(ii) $\mathrm{P}(\mathrm{e})$ is an intuitionistic neutrosophic subset of $\mathrm{Q}(\mathrm{e})$. $\mathrm{T}_{\mathrm{P}(\mathrm{e})}(\mathrm{x})$,

Or $\mathrm{T}_{\mathrm{P}(\mathrm{e})}(\mathrm{x}) \leq \mathrm{T}_{\mathrm{Q}(\mathrm{e})}(\mathrm{x}), \mathrm{I}_{\mathrm{P}(\mathrm{e})}(\mathrm{x}) \geq \mathrm{I}_{\mathrm{Q}(\mathrm{e})}(\mathrm{x}), \mathrm{F}_{\mathrm{P}(\mathrm{e})}(\mathrm{x}) \geq$

$\mathrm{F}_{\mathrm{Q}(\mathrm{e})}(\mathrm{x}), \forall \mathrm{e} \in \mathrm{A}, \mathrm{x} \in \mathrm{U}$.

We denote this relationship by $(\mathrm{P}, \mathrm{A}) \subseteq(\mathrm{Q}, \mathrm{B})$.

$(\mathrm{P}, \mathrm{A})$ is said to be intuitionistic neutrosophic soft super set of $(\mathrm{Q}, \mathrm{B})$ if $(\mathrm{Q}, \mathrm{B})$ is an intuitionistic neutrosophic soft subset of $(\mathrm{P}, \mathrm{A})$. We denote it by $(\mathrm{P}, \mathrm{A}) \supseteq(\mathrm{Q}, \mathrm{B})$.

\subsection{Definition [28]}

Two INSSs ( P, A ) and ( Q, B ) over the common universe $\mathrm{U}$ are said to be equal if $(\mathrm{P}, \mathrm{A})$ is an intuitionistic neutrosophic soft subset of $(\mathrm{Q}, \mathrm{B})$ and $(\mathrm{Q}, \mathrm{B})$ is an intuitionistic neutrosophic soft subset of $(\mathrm{P}, \mathrm{A})$ which can be denoted by $(\mathrm{P}, \mathrm{A})=(\mathrm{Q}, \mathrm{B})$.

\subsection{Definition [28]}

Let $(\mathrm{P}, \mathrm{A})$ and $(\mathrm{Q}, \mathrm{B})$ be two INSSs over the same universe $\mathrm{U}$. Then the union of $(\mathrm{P}, \mathrm{A})$ and $(\mathrm{Q}, \mathrm{B})$ is denoted by ' $(\mathrm{P}, \mathrm{A}) \cup(\mathrm{Q}, \mathrm{B})$ ' and is defined by $(\mathrm{P}, \mathrm{A}) \cup(\mathrm{Q}, \mathrm{B})=(\mathrm{K}$, $\mathrm{C}$, where $\mathrm{C}=\mathrm{A} \cup \mathrm{B}$ and the truth-membership, indeterminacy-membership and falsity-membership of ( $\mathrm{K}$,
C) are as follows:

$$
\begin{gathered}
\mathrm{T}_{\mathrm{K}(\varepsilon)}(\mathrm{m})=\left\{\begin{array}{c}
\mathrm{T}_{\mathrm{P}(\varepsilon)}(\mathrm{m}), \text { if } \varepsilon \in \mathrm{A}-\mathrm{B} \\
\mathrm{T}_{\mathrm{Q}(\varepsilon)}(\mathrm{m}), \text { if } \varepsilon \in \mathrm{B}-\mathrm{A} \\
\max \left(\mathrm{T}_{\mathrm{P}(\varepsilon)}(\mathrm{m}), \mathrm{T}_{\mathrm{Q}(\varepsilon)}(\mathrm{m})\right), \text { if } \varepsilon \in \mathrm{A} \cap \mathrm{B}
\end{array}\right. \\
\mathrm{I}_{\mathrm{K}(\varepsilon)}(\mathrm{m})=\left\{\begin{array}{c}
\mathrm{I}_{\mathrm{P}(\varepsilon)}(\mathrm{m}), \text { if } \varepsilon \in \mathrm{A}-\mathrm{B}, \\
\mathrm{I}_{\mathrm{Q}(\varepsilon)}(\mathrm{m}), \text { if } \varepsilon \in \mathrm{B}-\mathrm{A} \\
\min \left(\mathrm{I}_{\mathrm{P}(\varepsilon)}(\mathrm{m}), \mathrm{I}_{\mathrm{Q}(\varepsilon)}(\mathrm{m})\right), \text { if } \varepsilon \in \mathrm{A} \cap \mathrm{B}
\end{array}\right. \\
\mathrm{F}_{\mathrm{K}(\varepsilon)}(\mathrm{m})=\left\{\begin{array}{c}
(\mathrm{m}), \text { if } \varepsilon \in \mathrm{A}-\mathrm{B}, \\
\mathrm{F}_{\mathrm{Q}(\varepsilon)}(\mathrm{m}), \text { if } \varepsilon \in \mathrm{B}-\mathrm{A} \\
\min \left(\mathrm{F}_{\mathrm{P}(\varepsilon)}(\mathrm{m}), \mathrm{F}_{\mathrm{Q}(\varepsilon)}(\mathrm{m})\right), \text { if } \varepsilon \in \mathrm{A} \cap \mathrm{B}
\end{array}\right.
\end{gathered}
$$

\subsection{Definition[28]}

Let $(\mathrm{P}, \mathrm{A})$ and $(\mathrm{Q}, \mathrm{B})$ be two INSSs over the same universe $U$ such that $A \cap B \neq 0$. Then the intersection of $(P$, $\mathrm{A})$ and $(\mathrm{Q}, \mathrm{B})$ is denoted by ' $(\mathrm{P}, \mathrm{A}) \cap(\mathrm{Q}, \mathrm{B})$ ' and is defined by $(\mathrm{P}, \mathrm{A}) \cap(\mathrm{Q}, \mathrm{B})=(\mathrm{L}, \mathrm{C})$, where $\mathrm{C}=\mathrm{A} \cap \mathrm{B}$ and the truth-membership, indeterminacy membership and falsity-membership of $(\mathrm{L}, \mathrm{C})$ are related to those of $(\mathrm{P}, \mathrm{A})$ and $(\mathrm{Q}, \mathrm{B})$ by:

$$
\begin{gathered}
\mathrm{T}_{\mathrm{L}(\varepsilon)}(\mathrm{m})=\left\{\begin{array}{c}
\mathrm{T}_{\mathrm{P}(\varepsilon)}(\mathrm{m}), \text { if } \varepsilon \in \mathrm{A}-\mathrm{B}, \\
\mathrm{T}_{\mathrm{Q}(\varepsilon)}(\mathrm{m}), \text { if } \varepsilon \in \mathrm{B}-\mathrm{A} \\
\min \left(\mathrm{T}_{\mathrm{P}(\varepsilon)}(\mathrm{m}), \mathrm{T}_{\mathrm{Q}(\varepsilon)}(\mathrm{m})\right), \text { if } \varepsilon \in \mathrm{A} \cap \mathrm{B}
\end{array}\right. \\
\mathrm{I}_{\mathrm{L}(\varepsilon)}(\mathrm{m})=\left\{\begin{array}{c}
\mathrm{P}_{\mathrm{P}(\varepsilon)}(\mathrm{m}), \text { if } \varepsilon \in \mathrm{A}-\mathrm{B}, \\
\mathrm{I}_{\mathrm{Q}(\varepsilon)}(\mathrm{m}), \text { if } \varepsilon \in \mathrm{B}-\mathrm{A} \\
\min \left(\mathrm{I}_{\mathrm{P}(\varepsilon)}(\mathrm{m}), \mathrm{I}_{\mathrm{Q}(\varepsilon)}(\mathrm{m})\right), \text { if } \varepsilon \in \mathrm{A} \cap \mathrm{B}
\end{array}\right. \\
\mathrm{F}_{\mathrm{P}(\varepsilon)}(\mathrm{m}), \text { if } \varepsilon \in \mathrm{A}-\mathrm{B}, \\
\left.\mathrm{F}_{\mathrm{Q}(\varepsilon)}(\mathrm{m}), \text { if } \varepsilon \in \mathrm{B}-\mathrm{A}\right)
\end{gathered}
$$

\subsection{Definition [27]}

Let $(\mathrm{P}, \mathrm{A})$ be a soft set. The set $\operatorname{Supp}(\mathrm{P}, \mathrm{A})=\{\mathrm{x} \in \mathrm{A} \mid \mathrm{P}(\mathrm{x}) \neq$ $\emptyset\}$ is called the support of the soft set (P,A). A soft set $(\mathrm{P}, \mathrm{A})$ is non-null if $\operatorname{Supp}(\mathrm{P}, \mathrm{A}) \neq \varnothing$.

\subsection{Definition [41]}

A fuzzy subset $\mu$ of a ring $\mathrm{R}$ is called a fuzzy subring of $\mathrm{R}$ (in Rosenfeld' sense), if for all $\mathrm{x}, \mathrm{y} \in \mathrm{R}$ the following requirements are met:

$$
\begin{aligned}
& \mu(\mathrm{x}-\mathrm{y}) \geq \min (\mu(\mathrm{x}), \mu(y)) \text { and } \\
& \mu(\mathrm{xy}) \geq \min (\mu(\mathrm{x}), \mu(y))
\end{aligned}
$$

\section{Intuitionistic Neutrosophic Soft Set over Ring}

In this section, we introduce the notions of intuitionistic neutrosophic soft set over ring and intuitionistic 
neutrosophic soft subring in Rosenfeld's sense and study some of their properties related to this notions.

Throughout this paper. Let $(\mathrm{R},+,$.$) be a ring . E be a$ parameter set and let $\mathrm{A} \subseteq \mathrm{E}$. For the sake of simplicity, we will denote the ring $(\mathrm{R},+,$.$) simply as \mathrm{R}$.

From now on, $\mathrm{R}$ denotes a commutative ring and all intuitionistic neutrosophic soft sets are considered over R.

\subsection{Definition}

Let $(\tilde{P}, \mathrm{~A})$ be an intuitionistic neutrosophic soft set. The set $\operatorname{Supp}(\tilde{P}, \mathrm{~A})=\{\varepsilon \in \mathrm{A} \mid \tilde{P}(\varepsilon) \neq \emptyset\}$ is called the support of the intuitionistic neutrosophic soft set $(\tilde{P}, \mathrm{~A})$.An intuitionistic neutrosophic soft set $(\tilde{P}, \mathrm{~A})$ is non-null if Supp $(\tilde{P}, \mathrm{~A}) \neq \emptyset$.

\subsection{Definition}

A pair $(\tilde{P}, \mathrm{~A})$ is called an intuitionistic neutrosophic soft set over ring, where $\tilde{P}$ is a mapping given by

$\tilde{P}: \mathrm{A} \rightarrow([0,1] \times[0,1] \times[0,1])^{R}, \tilde{P}(\varepsilon): \mathrm{R} \rightarrow[0,1] \times$ $[0,1] \times[0,1]$

$\tilde{P}(\varepsilon)=\left\{\left(x, T_{\tilde{P}(\varepsilon)}(x), I_{\tilde{P}(\varepsilon)}(x), F_{\tilde{P}(\varepsilon)}(x)\right): x \in R\right\}$ for all $\varepsilon \in \mathrm{A}$,

If for all $\mathrm{x}, \mathrm{y} \in \mathrm{R}$ the following condition hold:

(1) $T_{\tilde{P}(\varepsilon)}(x-y) \geq T_{\tilde{P}(\varepsilon)}(x) \wedge T_{\tilde{P}(\varepsilon)}(y)$, $F_{\tilde{P}(\varepsilon)}(x-y) \leq F_{\tilde{P}(\varepsilon)}(x) \vee F_{\tilde{P}(\varepsilon)}(y)$ and $I_{\tilde{P}(\varepsilon)}(x-y) \leq I_{\tilde{P}(\varepsilon)}(x) \vee I_{\tilde{P}(\varepsilon)}(y)$

(2) $T_{\tilde{P}(\varepsilon)}(x y) \geq T_{\tilde{P}(\varepsilon)}(x) \wedge T_{\tilde{P}(\varepsilon)}(y)$, $F_{\tilde{P}(\varepsilon)}(x y) \leq F_{\tilde{P}(\varepsilon)}(x) \vee F_{\tilde{P}(\varepsilon)}(y)$ and $I_{\tilde{P}(\varepsilon)}(x y) \leq I_{\tilde{P}(\varepsilon)}(x) \vee I_{\tilde{P}(\varepsilon)}(y)$

\subsection{Definition}

For two intuitionistic neutrosophic soft set over ring $(\tilde{P}, \mathrm{~A})$ and $(\tilde{Q}, \mathrm{~B})$, we say that $(\tilde{P}, \mathrm{~A})$ is an intuitionistic neutrosophic soft subring of $(\tilde{Q}, \mathrm{~B})$ and write $(\tilde{P}, \mathrm{~A}) \subseteq$ $(\tilde{Q}, \mathrm{~B}) \quad$ if

(i) $\mathrm{A} \subseteq \mathrm{B}$

(ii) for each $\mathrm{x} \in R, \varepsilon \in \mathrm{A}, T_{\tilde{P}(\varepsilon)}(x) \leq T_{\tilde{Q}(\varepsilon)}(x)$,

$$
I_{\tilde{P}(\varepsilon)}(x) \geq I_{\tilde{Q}(\varepsilon)}(x), F_{\tilde{P}(\varepsilon)}(x) \geq F_{\tilde{Q}(\varepsilon)}(x) \cdot(\mathbf{8})
$$

\subsection{Definition}

Two intuitionist neutrosophic soft set over ring $(\widetilde{P}, \mathrm{~A})$ and $(\tilde{Q}, \mathrm{~B})$ are said to be equal if $(\tilde{P}, \mathrm{~A}) \subseteq(\tilde{Q}, \mathrm{~B})$ and $(\tilde{Q}, \mathrm{~B})$ $\subseteq(\tilde{P}, \mathrm{~A})$.

\subsection{Theorem}

Let $(\tilde{P}, \mathrm{~A})$ and $(\tilde{Q}, \mathrm{~B})$ be two intuitionistic neutrosophic soft over ring R. if $\tilde{P}(\varepsilon) \leq \tilde{Q}(\varepsilon)$ for all $\varepsilon \in \mathrm{A}$ and $\mathrm{A} \subset$ $\mathrm{B}$, then $(\tilde{P}, \mathrm{~A})$ is an intuitionistic neutrosophic soft subring of $(\tilde{Q}, \mathrm{~B})$.
Proof The proof is straightforward

\subsection{Definition}

The union of two intuitionistic neutrosophic soft set over $\operatorname{ring}(\tilde{P}, \mathrm{~A})$ and $(\tilde{Q}, \mathrm{~B})$ is denoted by $(\tilde{P}, \mathrm{~A}) \tilde{U}(\tilde{Q}, \mathrm{~B})$ and is defined by a intuitionistic neutrosophic soft set over ring

$\widetilde{H}: \mathrm{A} \cup \mathrm{B} \rightarrow([0,1] \times[0,1] \times[0,1])^{R}$ such that for each $\varepsilon \in \mathrm{A} \cup \mathrm{B}$.

$$
\begin{aligned}
& \widetilde{H}(\varepsilon)= \\
& \left\{\begin{array}{c}
<x, T_{\tilde{P}(\varepsilon)}(x), I_{\tilde{P}(\varepsilon)}(x), F_{\tilde{P}(\varepsilon)}(x)>\text { if } \varepsilon \in \mathrm{A}-\mathrm{B} \\
<x, T_{\tilde{Q}(\varepsilon)}(x), I_{\tilde{Q}(\varepsilon)}(x), F_{\tilde{Q}(\varepsilon)}(x)>\text { if } \varepsilon \in \mathrm{B}-\mathrm{A} \\
<x, T_{\tilde{P}(\varepsilon)}(x) \vee T_{\tilde{Q}(\varepsilon)}(x), I_{\tilde{P}(\varepsilon)}(x) \wedge I_{\tilde{Q}(\varepsilon)}(x), F_{\tilde{P}(\varepsilon)}(x) \wedge F_{\tilde{Q}(\varepsilon)}(x)>, \\
\text { if } \varepsilon \in \mathrm{A} \cap \mathrm{B}
\end{array}\right.
\end{aligned}
$$

This is denoted by $(\widetilde{H}, C)=(\widetilde{P}, \mathrm{~A}) \widetilde{U}(\tilde{Q}, \mathrm{~B})$, where $\mathrm{C}=\mathrm{A} \cup \mathrm{B}$.

\subsection{Theorem}

If $(\tilde{P}, \mathrm{~A})$ and $(\tilde{Q}, \mathrm{~B})$ are two intuitionistic neutrosophic soft set over ring $\mathrm{R}$, then, so are $(\tilde{P}, \mathrm{~A}) \widetilde{U}(\tilde{Q}, \mathrm{~B})$.

Proof. For any $\varepsilon \in A \cup B$ and $x, y \in R$, we consider the following cases.

Case 1. Let $\varepsilon \in \mathrm{A}-\mathrm{B}$.Then,

$$
\begin{aligned}
T_{\widetilde{H}(\varepsilon)}(x-y) & =T_{\tilde{P}(\varepsilon)}(x-y) \\
& \geq T_{\tilde{P}(\varepsilon)}(x) \wedge T_{\tilde{P}(\varepsilon)}(y) \\
& =T_{\tilde{H}(\varepsilon)}(x) \wedge T_{\tilde{H}(\varepsilon)}(y), \\
T_{\widetilde{H}(\varepsilon)}(x y)= & T_{\tilde{P}(\varepsilon)}(x y) \\
\geq & T_{\tilde{P}(\varepsilon)}(x) \wedge T_{\tilde{P}(\varepsilon)}(y) \\
= & T_{\tilde{H}(\varepsilon)}(x) \wedge T_{\tilde{H}(\varepsilon)}(y), \\
I_{\widetilde{H}(\varepsilon)}(x-y) & =I_{\tilde{P}(\varepsilon)}(x-y) \\
\leq & I_{\tilde{P}(\varepsilon)}(x) \vee I_{\tilde{P}(\varepsilon)}(y) \\
= & I_{\tilde{H}(\varepsilon)}(x) \vee I_{\tilde{H}(\varepsilon)}(y), \\
I_{\widetilde{H}(\varepsilon)}(x y)= & I_{\tilde{P}(\varepsilon)}(x y) \\
\leq & I_{\tilde{P}(\varepsilon)}(x) \vee I_{\tilde{P}(\varepsilon)}(y) \\
= & I_{\widetilde{H}(\varepsilon)}(x) \vee I_{\widetilde{H}(\varepsilon)}(y), \\
F_{\widetilde{H}(\varepsilon)}(x-y) & =F_{\tilde{P}(\varepsilon)}(x-y) \\
& \leq F_{\tilde{P}(\varepsilon)}(x) \vee F_{\tilde{P}(\varepsilon)}(y) \\
& =F_{\tilde{H}(\varepsilon)}(x) \vee F_{\tilde{H}(\varepsilon)}(y), \\
F_{\widetilde{H}(\varepsilon)}(x y)= & F_{\tilde{P}(\varepsilon)}(x y) \\
\leq & F_{\tilde{P}(\varepsilon)}(x) \vee F_{\tilde{P}(\varepsilon)}(y) \\
= & F_{\tilde{H}(\varepsilon)}(x) \vee F_{\tilde{H}(\varepsilon)}(y),
\end{aligned}
$$

Case 2. Let if $\varepsilon \in \mathrm{B}-\mathrm{A}$. Then, analogous to the proof of case 1 , we have

$$
\begin{aligned}
& T_{\widetilde{H}(\varepsilon)}(x-y) \geq T_{\widetilde{H}(\varepsilon)}(x) \wedge T_{\widetilde{H}(\varepsilon)}(y) \\
& T_{\overparen{H}(\varepsilon)}(x y) \geq T_{\widetilde{H}(\varepsilon)}(x) \wedge T_{\widetilde{H}(\varepsilon)}(y) \\
& I_{\widetilde{H}(\varepsilon)}(x-y) \leq I_{\widetilde{H}(\varepsilon)}(x) \vee I_{\widetilde{H}(\varepsilon)}(y) \\
& I_{\overparen{H}(\varepsilon)}(x y) \leq I_{\widetilde{H}(\varepsilon)}(x) \vee I_{\widetilde{H}(\varepsilon)}(y)
\end{aligned}
$$




$$
\begin{aligned}
& F_{\widetilde{H}(\varepsilon)}(x-y) \leq F_{\widetilde{H}(\varepsilon)}(x) \vee F_{\widetilde{H}(\varepsilon)}(y) \\
& F_{H(\varepsilon)}(x y) \leq F_{H(\varepsilon)}(x) \vee F_{H(\varepsilon)}(y)
\end{aligned}
$$

Case 3. Let $\varepsilon \in A \cap B$. In this case the proof is straightforward. thus, in any cases, we have

$$
\begin{aligned}
& T_{\widetilde{H}(\varepsilon)}(x-y) \geq T_{\widetilde{H}(\varepsilon)}(x) \wedge T_{\widetilde{H}(\varepsilon)}(y) \\
& T_{\widetilde{H}(\varepsilon)}(x y) \geq T_{\widetilde{H}(\varepsilon)}(x) \wedge T_{\widetilde{H}(\varepsilon)}(y) \\
& I_{\widetilde{H}(\varepsilon)}(x-y) \leq I_{\widetilde{H}(\varepsilon)}(x) \vee I_{\widetilde{H}(\varepsilon)}(y) \\
& I_{\widetilde{H}(\varepsilon)}(x y) \leq I_{\widetilde{H}(\varepsilon)}(x) \vee I_{\widetilde{H}(\varepsilon)}(y) \\
& F_{\widetilde{H}(\varepsilon)}(x-y) \leq F_{\widetilde{H}(\varepsilon)}(x) \vee F_{\widetilde{H}(\varepsilon)}(y) \\
& F_{\widetilde{H}(\varepsilon)}(x y) \leq F_{\widetilde{H}(\varepsilon)}(x) \vee F_{\widetilde{H}(\varepsilon)}(y)
\end{aligned}
$$

Therefore, $(\tilde{P}, \mathrm{~A}) \widetilde{U}(\tilde{Q}, \mathrm{~B})$ is an intuitionistic neutrosophic soft set over ring

\subsection{Definition}

The intersection of two intuitionistic neutrosophic soft set over ring $(\widetilde{P}, \mathrm{~A})$ and $(\widetilde{Q}, \mathrm{~B})$ is denoted by $(\tilde{P}, \mathrm{~A}) \widetilde{\cap}(\widetilde{Q}, \mathrm{~B})$ and is defined by an intuitionistic neutrosophic soft set over ring.

$\widetilde{M}: \mathrm{A} \cup \mathrm{B} \rightarrow([0,1] \times[0,1] \times[0,1])^{R}$ such that for each $\varepsilon \in \mathrm{A} U \mathrm{~B}$.

$$
\begin{aligned}
& \widetilde{M}(\varepsilon)= \\
& \left\{\begin{array}{c}
<x, T_{\tilde{P}(\varepsilon)}(x), I_{\tilde{P}(\varepsilon)}(x), F_{\tilde{P}(\varepsilon)}(x)>\text { if } \varepsilon \in \mathrm{A}-\mathrm{B} \\
<x, T_{\tilde{Q}(\varepsilon)}(x), I_{\tilde{Q}(\varepsilon)}(x), F_{\tilde{Q}(\varepsilon)}(x)>\text { if } \varepsilon \in \mathrm{B}-\mathrm{A} \\
<x, T_{\tilde{P}(\varepsilon)}(x) \wedge T_{\tilde{Q}(\varepsilon)}(x), I_{\tilde{P}(\varepsilon)}(x) \wedge I_{\tilde{Q}(\varepsilon)}(x), F_{\tilde{P}(\varepsilon)}(x) \vee F_{\tilde{Q}(\varepsilon)}(x)>, \\
\text { if } \varepsilon \in \mathrm{A} \cap \mathrm{B}
\end{array}\right.
\end{aligned}
$$

This is denoted by $(\widetilde{M}, C)=(\widetilde{P}, \mathrm{~A}) \widetilde{\cap}(\widetilde{Q}, \mathrm{~B})$, where $\mathrm{C}=$ $\mathrm{AU}$ B .

\subsection{Theorem}

If $(\tilde{P}, \mathrm{~A})$ and $(\tilde{Q}, \mathrm{~B})$ are two intuitionistic neutrosophic soft set over ring, then, so are $(\tilde{P}, \mathrm{~A}) \widetilde{\cap}(\widetilde{Q}, \mathrm{~B})$.

Proof. The proof is similar to that of Theorem 3.8.

\subsection{Definition}

Let $(\tilde{P}, \mathrm{~A})$ and $(\tilde{Q}, \mathrm{~B})$ be two intuitionistic neutrosophic soft set over ring $\mathrm{R}$. Then , " $(\tilde{P}, \mathrm{~A})$ AND $(\tilde{Q}, \mathrm{~B})$ " is denoted by $(\tilde{P}, \mathrm{~A}) \tilde{\Lambda}(\tilde{Q}, \mathrm{~B})$ and is defined by $(\tilde{P}, \mathrm{~A}) \tilde{\Lambda}(\tilde{Q}, \mathrm{~B})=$ $(\widetilde{N}, C)$, where $\mathrm{C}=\mathrm{A} \times \mathrm{B}$ and $\widetilde{H}: \mathrm{C} \rightarrow\left([0,1]^{3} \times[0,1]^{3}\right)^{R}$ is defined as

$$
\widetilde{N}(\alpha, \beta)=\tilde{P}(\alpha) \cap \tilde{Q}(\beta), \text { for all }(\alpha, \beta) \in \mathrm{C} .
$$

\subsection{Theorem}

If $(\tilde{P}, \mathrm{~A})$ and $(\tilde{Q}, \mathrm{~B})$ are two intuitionistic neutrosophic soft set over ring $\mathrm{R}$, then, so is $(\tilde{P}, \mathrm{~A}) \tilde{\Lambda}(\tilde{Q}, \mathrm{~B})$.

Proof. For all $\mathrm{x}, \mathrm{y} \in \mathrm{R}$ and $(\alpha, \beta) \in \mathrm{A} \mathrm{x} \mathrm{B}$ we have

$$
\begin{aligned}
& T_{\tilde{N}(\alpha, \beta)}(x-y)=\left(T_{\tilde{P}(\alpha)}(x-y) \wedge T_{\tilde{Q}(\beta)}(x-y)\right) \\
& \quad \geq\left(T_{\tilde{P}(\alpha)}(x) \wedge T_{\tilde{P}(\alpha)}(y)\right) \wedge\left(T_{\tilde{Q}(\beta)}(x) \wedge T_{\tilde{Q}(\beta)}(y)\right) \\
& \quad=\left(T_{\tilde{P}(\alpha)}(x) \wedge T_{\tilde{Q}(\beta)}(x)\right) \wedge\left(T_{\tilde{P}(\alpha)}(y) \wedge T_{\tilde{Q}(\beta)}(y)\right) \\
& \quad=T_{\widetilde{N}(\alpha, \beta)}(x) \wedge T_{\widetilde{N}(\alpha, \beta)}(y) \\
& T_{\widetilde{N}(\alpha, \beta)}(x y)=\left(T_{\tilde{P}(\alpha)}(x y) \wedge T_{\tilde{Q}(\beta)}(x y)\right) \\
& \quad \geq\left(T_{\tilde{P}(\alpha)}(x) \wedge T_{\tilde{P}(\alpha)}(y)\right) \wedge\left(T_{\tilde{Q}(\beta)}(x) \wedge T_{\tilde{Q}(\beta)}(y)\right) \\
& =\left(T_{\tilde{P}(\alpha)}(x) \wedge T_{\tilde{Q}(\beta)}(x)\right) \wedge\left(T_{\tilde{P}(\alpha)}(y) \wedge T_{\tilde{Q}(\beta)}(y)\right) \\
& =T_{\widetilde{N}(\alpha, \beta)}(x) \wedge T_{\widetilde{N}(\alpha, \beta)}(y)
\end{aligned}
$$

In a similar way, we have

$I_{\widetilde{N}(\alpha, \beta)}(x-y) \leq I_{\widetilde{N}(\alpha, \beta)}(x) \vee I_{\widetilde{N}(\alpha, \beta)}(y)$

$I_{\widetilde{N}(\alpha, \beta)}(x y) \leq I_{\widetilde{N}(\alpha, \beta)}(x) \vee I_{\widetilde{N}(\alpha, \beta)}(y)$

$F_{\tilde{N}(\alpha, \beta)}(x-y) \leq F_{\tilde{N}(\alpha, \beta)}(x) \vee F_{\tilde{N}(\alpha, \beta)}(y)$

$F_{\tilde{N}(\alpha, \beta)}(x y) \leq F_{\tilde{N}(\alpha, \beta)}(x) \vee F_{\tilde{N}(\alpha, \beta)}(y)$

For all $\mathrm{x}, \mathrm{y} \in \mathrm{R}$ and $(\alpha, \beta) \in \mathrm{C}$.It follows that $(\tilde{P}, \mathrm{~A}) \tilde{\wedge}(\tilde{Q}, \mathrm{~B})$ is an intuitionistic neutrosophic soft set over ring $\mathrm{R}$.

\subsection{Definition}

Let $(\tilde{P}, \mathrm{~A})$ and $(\tilde{Q}, \mathrm{~B})$ be two intuitionistic neutrosophic soft set over ring R. Then, "( $\widetilde{P}, \mathrm{~A})$ OR $(\tilde{Q}, \mathrm{~B})$ " is denoted by $(\tilde{P}, \mathrm{~A}) \quad \widetilde{V}(\tilde{Q}, \mathrm{~B})$ and is defined by $(\tilde{P}, \mathrm{~A})$ $\widetilde{\mathrm{V}}(\tilde{Q}, \mathrm{~B})=(\tilde{O}, C)$, where $\mathrm{C}=\mathrm{A} \times \mathrm{B}$ and $\tilde{O}: \mathrm{C} \rightarrow$ $\left([0,1]^{3} \times[0,1]^{3}\right)^{R}$ is defined as

$$
\tilde{O}(\alpha, \beta)=\tilde{P}(\alpha) \widetilde{\cup} \tilde{Q}(\beta), \text { for all }(\alpha, \beta) \in \mathrm{C} .
$$

\subsection{Theorem}

If ( $\tilde{P}, A)$ and ( $\tilde{Q}, \mathrm{~B})$ are two intuitionist neutrosophic soft set over ring $\mathrm{R}$, then, so are $(\tilde{P}, \mathrm{~A}) \widetilde{\mathrm{V}}(\tilde{Q}, \mathrm{~B})$.

Proof. The proof is straightforward.

The following theorem is a generalization of previous results.

\subsection{Theorem}

Let $(\tilde{P}, \mathrm{~A})$ be an intuitionist neutrosophic soft set over ring $\mathrm{R}$, and let $\left\{\left(\tilde{P}_{i}, A_{i}\right)\right\}_{i \in I}$ be a nonempty family of intuitionistic neutrosophic soft set over ring, where $\mathrm{I}$ is an index set .Then, one has the following:

(1) $\wedge_{i \in I}\left(\tilde{P}_{i}, A_{i}\right)$ is an intuitionistic neutrosophic soft set over ring R.

(2) if $A_{i} \cap A_{j}=0$, for all $\mathrm{i}, \mathrm{j} \in \mathrm{I}$, then $\bigvee_{i \in I}\left(\tilde{P}_{i}, A_{i}\right)$ is an intuitionistic neutrosophic soft set over ring $\mathrm{R}$.

\subsection{Definition}

Let $(\tilde{P}, \mathrm{~A})$ and $(\tilde{Q}, \mathrm{~B})$ be two intuitionistic neutrosophic 
soft set over ring $\mathrm{R}$. Then ,the product of $(\tilde{P}, \mathrm{~A})$ and $(\tilde{Q}, \mathrm{~B})$ is defined to be the intuitionistic neutrosophic soft set over ring $(\tilde{P} \circ \tilde{Q}, \mathrm{C})$, where $\mathrm{C}=\mathrm{A} \cup \mathrm{B}$ and

$$
\begin{aligned}
& T_{(\tilde{P} \circ \tilde{Q})(\varepsilon)}(x) \\
& =\left\{\begin{array}{c}
T_{\tilde{P}(\varepsilon)}(x) \text { if } \varepsilon \in \mathrm{A}-\mathrm{B} \\
T_{\tilde{Q}(\varepsilon)}(x) \text { if } \varepsilon \in \mathrm{B}-\mathrm{A} \\
\mathrm{V}_{x=a b}\left\{T_{\tilde{P}(\varepsilon)}(a) \wedge T_{\tilde{Q}(\varepsilon)}(b)\right\} \text { if } \varepsilon \in \mathrm{A} \cap \mathrm{B}
\end{array}\right. \\
& I_{(\tilde{P} \circ \tilde{Q})(\varepsilon)}(x)=\left\{\begin{array}{c}
I_{\tilde{P}(\varepsilon)}(x) \text { if } \varepsilon \in \mathrm{A}-\mathrm{B} \\
I_{\tilde{Q}(\varepsilon)}(x) \text { if } \varepsilon \in \mathrm{B}-\mathrm{A} \\
\Lambda_{x=a b}\left\{I_{\tilde{P}(\varepsilon)}(a) \vee I_{\tilde{Q}(\varepsilon)}(b)\right\} \text { if } \varepsilon \in \mathrm{A} \cap \mathrm{B}
\end{array}\right. \\
& F_{(\tilde{P} \circ \tilde{Q})(\varepsilon)}(x) \\
& =\left\{\begin{array}{c}
F_{\tilde{P}(\varepsilon)}(x) \text { if } \varepsilon \in \mathrm{A}-\mathrm{B} \\
F_{\tilde{Q}(\varepsilon)}(x) \text { if } \varepsilon \in \mathrm{B}-\mathrm{A} \\
\Lambda_{x=a b}\left\{F_{\tilde{P}(\varepsilon)}(a) \vee F_{\tilde{Q}(\varepsilon)}(b)\right\} \text { if } \varepsilon \in \mathrm{A} \cap \mathrm{B}, \mathrm{a}, \mathrm{b} \in R
\end{array}\right.
\end{aligned}
$$

For all $\varepsilon \in \mathrm{C}$ and $\mathrm{a}, \mathrm{b} \in \mathrm{R}$. This is denoted by $(\tilde{P} \circ \tilde{Q}, \mathrm{C})$ $=(\tilde{P}, \mathrm{~A}) \circ(\tilde{Q}, \mathrm{~B})$.

\subsection{Theorem}

If $(\tilde{P}, \mathrm{~A})$ and $(\tilde{Q}, \mathrm{~B})$ are two intuitionistic neutrosophic soft set over ring $\mathrm{R}$. Then, so is $(\tilde{P}, \mathrm{~A}) \circ(\tilde{Q}, \mathrm{~B})$.

Proof. Let $(\tilde{P}, \mathrm{~A})$ and $(\tilde{Q}, \mathrm{~B})$ be two intuitionistic neutrosophic soft set over ring $\mathrm{R}$. Then ,for any $\varepsilon \in A U$ $\mathrm{B}$, and $\mathrm{x}, \mathrm{y} \in \mathrm{R}$, we consider the following cases.

Case 1. Let $\varepsilon \in A-B$. Then,

$$
\begin{aligned}
T_{(\tilde{P} \circ \tilde{Q})(\varepsilon)}(x-y) & =T_{\tilde{P}(\varepsilon)}(x-y) \\
& \geq T_{\tilde{P}(\varepsilon)}(x) \wedge T_{\tilde{P}(\varepsilon)}(y) \\
= & T_{(\tilde{P} \circ \tilde{Q})(\varepsilon)}(x) \wedge T_{(\tilde{P} \circ \tilde{Q})(\varepsilon)}(y), \\
T_{(\tilde{P} \circ \tilde{Q})(\varepsilon)}(x y)= & T_{\tilde{P}(\varepsilon)}(x y) \\
\geq & T_{\tilde{P}(\varepsilon)}(x) \wedge T_{\tilde{P}(\varepsilon)}(y) \\
= & T_{(\tilde{P} \circ \tilde{Q})(\varepsilon)}(x) \wedge T_{(\tilde{P} \circ \tilde{Q})(\varepsilon)}(y) \\
I_{(\tilde{P} \circ \tilde{Q})(\varepsilon)}(x-y) & =I_{\tilde{P}(\varepsilon)}(x-y) \\
& \leq I_{\tilde{P}(\varepsilon)}(x) \vee I_{\tilde{P}(\varepsilon)}(y) \\
& =I_{(\tilde{P} \circ \tilde{Q})(\varepsilon)}(x) \vee I_{(\tilde{P} \circ \tilde{Q})(\varepsilon)}(y), \\
I_{(\tilde{P} \circ \tilde{Q})(\varepsilon)}(x y)= & I_{\tilde{P}(\varepsilon)}(x y) \\
\leq & I_{\tilde{P}(\varepsilon)}(x) \vee I_{\tilde{P}(\varepsilon)}(y) \\
= & I_{(\tilde{P} \circ \tilde{Q})(\varepsilon)}(x) \vee I_{(\tilde{P} \circ \tilde{Q})(\varepsilon)}(y) \\
F_{(\tilde{P} \circ \tilde{Q})(\varepsilon)}(x-y) & =F_{\tilde{P}(\varepsilon)}(x-y) \\
& \leq F_{\tilde{P}(\varepsilon)}(x) \vee F_{\tilde{P}(\varepsilon)}(y) \\
& =F_{(\tilde{P} \circ \tilde{Q})(\varepsilon)}(x) \vee F_{(\tilde{P} \circ \tilde{Q})(\varepsilon)}(y), \\
F_{(\tilde{P} \circ \tilde{Q})(\varepsilon)}(x y)= & F_{\tilde{P}(\varepsilon)}(x y) \\
& \leq F_{\tilde{P}(\varepsilon)}(x) \vee F_{\tilde{P}(\varepsilon)}(y) \\
& =F_{(\tilde{P} \circ \tilde{Q})(\varepsilon)}(x) \vee F_{(\tilde{P} \circ \tilde{Q})(\varepsilon)}(y)
\end{aligned}
$$

Case 2. Let $\varepsilon \in \mathrm{B}-\mathrm{A}$. Then, analogous to the proof of case 1,the proof is straightforward.

Case 3. Let $\varepsilon \in A \cap B$. Then,

$T_{(\tilde{P} \circ \tilde{Q})(\varepsilon)}(x)=\bigvee_{x=a b}\left(T_{\tilde{P}(\varepsilon)}(a) \wedge T_{\tilde{Q}(\varepsilon)}(b)\right)$

$$
\begin{aligned}
& \geq \mathrm{V}_{x y=a b y}\left(T_{\tilde{P}(\varepsilon)}(a) \wedge T_{\tilde{Q}(\varepsilon)}(b y)\right) \\
& \geq \mathrm{V}_{x y=c d}\left(T_{\tilde{P}(\varepsilon)}(c) \wedge T_{\tilde{Q}(\varepsilon)}(d)\right) \\
& =T_{(\tilde{P} \circ \tilde{Q})(\varepsilon)}(x y)
\end{aligned}
$$

Similarly, we have $T_{(\tilde{P} \circ \tilde{Q})(\varepsilon)}(x y) \geq T_{(\tilde{P} \circ \tilde{Q})(\varepsilon)}(y)$, and so

$T_{(\tilde{P} \circ \tilde{Q})(\varepsilon)}(x y) \geq T_{(\tilde{P} \circ \tilde{Q})(\varepsilon)}(x) \wedge T_{(\tilde{P} \circ \tilde{Q})(\varepsilon)}(y)$

In a similar way, we prove that

$I_{(\tilde{P} \circ \tilde{Q})(\varepsilon)}(x y) \leq I_{(\tilde{P} \circ \tilde{Q})(\varepsilon)}(x) \vee I_{(\tilde{P} \circ \tilde{Q})(\varepsilon)}(y)$

and $F_{(\tilde{P} \circ \tilde{Q})(\varepsilon)}(x y) \leq F_{(\tilde{P} \circ \tilde{Q})(\varepsilon)}(x) \vee F_{(\tilde{P} \circ \tilde{Q})(\varepsilon)}(y)$

Therefore $(\tilde{P}, \mathrm{~A}) \circ(\tilde{Q}, \mathrm{~B})$ is an intuitionistic neutrosophic soft set over ring $\mathrm{R}$.

\section{Conclusion}

In this paper we have introduced the concept of intuitionistic neutrosophic soft set over ring (INSSOR for short ). We also studied and discussed some properties related to this concept. The definitions of intersection, union, AND, and OR operations over ring (INSSOR) have also been defined. we have defined the product of two intuitionistic neutrosophic soft set over ring. Finally, it is hoped that this concept will be useful for the researchers to further promote and advance the research in neutrosophic soft set theory.

\section{Acknowledgments}

The authors are highly grateful to the referees for their valuable comments and suggestions for improving the paper.

\section{REFERENCES}

[1] L.A.Zadeh," Fuzzy sets". Information and Control.(1965), 8: pp.338-353.

[2] K. Atanassov," Intuitionistic fuzzy sets". Fuzzy Sets and Systems.(1986), 20, pp.87-96.

[3] Turksen, "Interval valued fuzzy sets based on normal forms". Fuzzy Sets and Systems, 20,(1968), pp.191-210.

[4] F.Smarandache ,"A Unifying Field in Logics. Neutrosophy: Neutrosophic Probability, Set and Logic". Rehoboth: American Research Press,(1998).

[5] M.Arora, R.Biswas,U.S.Pandy, "Neutrosophic Relational Database Decomposition", International Journal of Advanced Computer Science and Applications, Vol. 2, No. 8, (2011), pp.121-125.

[6] M. Arora and R. Biswas," Deployment of Neutrosophic technology to retrieve answers for queries posed in natural

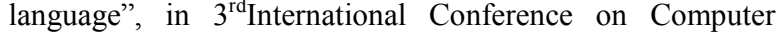
Science and Information Technology ICCSIT, IEEE catalog Number CFP1057E-art,Vol.3, ISBN:978-1-4244-5540-9,(20 $10)$, pp. 435-439. 
[7] Ansari, Biswas, Aggarwal,"Proposal for Applicability of Neutrosophic Set Theory in Medical AI", International Journal of Computer Applications (0975 - 8887), Vo 27No.5, (2011), pp:5-11

[8] A. Kharal, "A Neutrosophic Multicriteria Decision Making Method ",New Mathematics \& Natural Computation, to appear in Nov 2013.

[9] F.G Lupiáñez, "On neutrosophic topology", Kybernetes, Vol. 37 Iss: 6,(2008), pp.797 - 800 ,Doi:10.1108/0368492081087 6990.

[10] S. Aggarwal, R. Biswas, A.Q.Ansari," Neutrosophic Modeling and Control", 978-1-4244-9034-/10/\$26.00@2010 IEEE, pp.718-723.

[11] M. Bhowmik and M. Pal ," Intuitionistic Neutrosophic Set", ISSN 1746-7659, England, UK, Journal of Information and Computing Science,Vol. 4, No. 2, (2009), pp. 142-152.

[12] M. Bhowmik and M. Pal," Intuitionistic Neutrosophic Set Relations and Some of Its Properties, ISSN 1746-7659, England, UK, Journal of Information and Computing Science, Vol. 5, No. 3, (2010), pp. 183-192

[13] A. A. Salama, S.A.Alblowi, "Generalized Neutrosophic Set and Generalized Neutrosophic TopologicalSpaces" ,Comput er Science and Engineering, p-ISSN: 2163-1484 e-ISSN: 2163-1492 DOI: 10.5923/j.computer.20120207.01,2(7), 2012), pp. 129-132

[14] Wang, H., Smarandache, F., Zhang, Y. Q., Sunderraman, R,"Single valued neutrosophic sets". Multispace and Multistructure, 4,(2010), pp. 410-413.

[15] D. A. Molodtsov, "Soft Set Theory - First Result", Computers and Mathematics with Applications, Vol. 37, (1999),pp. 19-31.

[16] P. K. Maji, R. Biswas and A.R. Roy, "Fuzzy Soft Sets", Journal of Fuzzy Mathematics, Vol 9 , no.3, (2001), pp. 589-602.

[17] B. Ahmad, and A. Kharal, On Fuzzy Soft Sets, Hindawi Publishing Corporation, Advances in Fuzzy Systems, volume Article ID 586507, (2009), 6 pages doi: $10.1155 / 2009 / 586507$.

[18] P. K. Maji, A. R. Roy and R. Biswas, "Fuzzy soft sets" ,Journal of Fuzzy Mathematics. 9 (3),(2001), pp.589-602.

[19] T. J. Neog and D. K. Sut, "On Fuzzy Soft Complement and Related Properties", Accepted for publication in International, Journal of Energy, Information and communications (IJEIC).

[20] M. Borah, T. J. Neog and D. K. Sut," A study on some operations of fuzzy soft sets", International Journal of Modern Engineering Research (IJMER), Vol.2, Issue. 2,(2012), pp. 157-168.

[21] H. L. Yang, "Notes On Generalized Fuzzy Soft Sets", Journal of Mathematical Research and Exposition, Vol 31, No. 3, (2011), pp.567-570.

[22] P. Majumdar, S. K. Samanta, "Generalized Fuzzy Soft Sets", Computers and Mathematics with Applications, 59, (2010), pp.1425-1432.

[23] S. Alkhazaleh, A. R. Salleh, and N. Hassan," Possibility Fuzzy Soft Set", Advances in Decision Sciences,Vol 2011, Article ID 479756,18pages, doi:10.1155/2011/479756.

[24] P. K. Maji, R. Biswas, A. R. Roy, "Intuitionistic fuzzy soft sets", The journal of fuzzy mathematics 9(3)( 2001 ), pp.677-692.

[25] K.V .Babitha and J. J. Sunil," Generalized Intuitionistic Fuzzy Soft Sets and Its Applications ",Gen. Math. Notes, ISSN 2219-7184; Copyright (C) ICSRS Publication, (2011), Vol. 7, No. 2, (2011), pp.1-14.

[26] M.Bashir, A.R. Salleh, and S. Alkhazaleh," Possibility Intuitionistic Fuzzy Soft Set", Advances in Decision Sciences Volume 2012 (2012), Article ID 404325, 24 pages, doi:10.1155/2012/404325.

[27] P. K. Maji," Neutrosophic Soft Set", Annals of Fuzzy Mathematics and Informatics, Vol 5, No. 1,ISSN: 2093-9310, ISSN: 2287-623.

[28] S.Broumi and F. Smarandache, "Intuitionistic Neutrosophic Soft Set", Journal of Information and Computing Science, England, UK ,ISSN 1746-7659,Vol. 8, No. 2, (2013), pp.130-140.

[29] S.Broumi, “Generalized Neutrosophic Soft Set”, International Journal of Computer Science, Engineering and Information Technology (IJCSEIT), ISSN: 2231-3605, E-ISSN : 2231-3117,Vol.3, No.2, (2013), pp.17-30.

[30] H. Aktas \& N. Cagman, "Soft Sets and Soft Groups", Inf. Sci 177 (13) (2007), pp.2726-2735.

[31] A. Aygunoglu \& H. Aygun, "Introduction to Fuzzy Soft Groups”, Comput. Math. Appl. 58 (2009), pp. 1279-1286.

[32] A. Rosenfeld, "Fuzzy Groups", J. Math. Anal. Appl. 35 (1971), pp.512-517.

[33] A. Sezgin \& A.O. Atagun, "Soft Groups and Normalistic Soft Groups", Computers and Mathematics with Applications 62 (2011), pp.685-698.

[34] U. Acar, F. Koyuncu \& B. Tanay, "Soft Sets and Soft Rings", Computers and Mathematics with Applications: doi:10.1016/j.camwa.2010.03.034 (2010).

[35] J. Ghosh, B. Dinda \& T.K. Samanta, "Fuzzy Soft Rings and Fuzzy Soft Ideals", Int. J. P. App. Sc. Tech. 2(2) (2011), pp.66-74.

[36] E. Inan \& M.A. Ozturk, "Fuzzy Soft Rings and Fuzzy Soft Ideals", Neural Computing and Applications: doi: 10.1007/s00521-011-0550-5 (2011).

[37] B.Varol, "on fuzzy soft ring”, Journal of Hyperstructures, IS SN:2251-8436, print:2322-1666 online, 1(2) 2012, pp.1-15.

[38] J. Zhan, X. Liu, D. Xiang, K. P. Shum,"Soft Related to Fuzzy Set Theory", Hacettepe Journal of Mathematics and Statistics ,Volume 42 (1) (2013), pp.51 - 66.

[39] G. Selvachandran and A. R. Salleh,"Vague Soft Rings and Vague Soft Ideals", International Journal of Algebra, Vol. 6, 2012, no. 12 , pp. $557-572$.

[40] Z. Zhang, "Intuitionistic Fuzzy Soft Rings", International Journal of Fuzzy Systems, Vol. 14, No. 3, September 2012, pp.420-433.

[41] WANG, X-P., MO, Z.W., and LIU, W.J., "Fuzzy ideals generated by fuzzy points in semigroups", J. Sichuan Normal Univ., 15, (1992), pp. 17-24 